\title{
A high level of IncFGD5-AS1 inhibits epithelial-to-Mesenchymal transition by regulating the miR-196a-5p/SMAD6/BMP axis in gastric Cancer
}

Lin Liu', Cheng Zhang ${ }^{1}$, Jizhao Wang ${ }^{1}$, Xu Liu' ${ }^{1}$ Hangying Qu', Guangjian Zhang ${ }^{1}$, Ting Liang ${ }^{2}$, Jiansheng Wang ${ }^{1 *}$ and Jia Zhang ${ }^{1 *}$

\begin{abstract}
Background: Long non-coding RNA (IncRNA) was a vital factor in the progression and initiation of human cancers. This study found a new IncRNA, FGD5-AS1, which can inhibit EMT process, proliferation, and metastasis in vitro and in vivo.

Methods: GRT-PCR was employed to test the expression of IncFGD5-AS1 in 30 gastric cancer patients' cancer tissue and para-cancer tissue. Overexpressed IncFGD5-AS1 cells shown sharply decrease of proliferation, migration, and epithelial-mesenchymal transition (EMT). miR-196a-5p/SMAD6 was confirmed as downstream molecular mechanism of IncFGD5-AS1 by expression correlation analysis and mechanism experiments. In vivo study illustrated overexpression of IncFGD5-AS1 suppression tumor growth.

Results: LncFGD5-AS1 served as a ceRNA of miR-196a-5p to release its inhibition on SMAD6, a conventional inhibitor on the BMP pathway. Comparing with normal gastric cancer cells, FGD5-AS1 overexpressed group had fewer migration cells, lower cell viability, and lower EMT transformation rate. Meanwhile, xenografts nude mice injecting with overexpressed-FGD5-AS1 cells also shown smaller tumor weight and volume.
\end{abstract}

Conclusion: In conclusion, this research supported the first evidence that FGD5-AS1 suppressed proliferation and metastasis in gastric cancer by regulating miR-196a-5p/SMAD6/BMP axis and suggested a potential therapeutic candidate for gastric cancer.

Keywords: IncFGD5-AS1 1, miR-196a-5p 2 , ceRNA 3 , Epithelial-mesenchymal transition ${ }_{4}$, Prognose of gastric Cancer

\section{Background}

According to the clinical research carried out in 2013, gastric cancer is the third most common and the third lethal tumor [1]. In various clinical research focused on mortality of gastric cancer, the metastasis markers pTNM, rN and positive lymph node ratio closely

\footnotetext{
* Correspondence: wangjsh@mail.xjtu.edu.cn; zhangjiaxjtu@mail.xjtu.edu.cn ${ }^{1}$ The Department of Thoracic Surgery, The First Affiliated Hospital of Xi'an Jiaotong University, Xi'an, Shaanxi, China

Full list of author information is available at the end of the article
}

correlate with poor prognosis and shorter survival. Among those who die of gastric cancer, metastatic gastric cancer accounts for majority [2-4]. The significantly high lethality rate of metastatic gastric cancer can be due to the concealment of metastasis. Until now, clinicians still lack appropriate methods to mitigate postmetastatic gastric cancer because current operative treatment only gets considerable success in the early stage of gastric cancer [5-7]. Based on these reasons, a proper

(c) The Author(s). 2021 Open Access This article is licensed under a Creative Commons Attribution 4.0 International License, which permits use, sharing, adaptation, distribution and reproduction in any medium or format, as long as you give appropriate credit to the original author(s) and the source, provide a link to the Creative Commons licence, and indicate if changes were made. The images or other third party material in this article are included in the article's Creative Commons licence, unless indicated otherwise in a credit line to the material. If material is not included in the article's Creative Commons licence and your intended use is not permitted by statutory regulation or exceeds the permitted use, you will need to obtain permission directly from the copyright holder. To view a copy of this licence, visit http://creativecommons.org/licenses/by/4.0/. The Creative Commons Public Domain Dedication waiver (http://creativecommons.org/publicdomain/zero/1.0/) applies to the data made available in this article, unless otherwise stated in a credit line to the data. 
treatment point of metastasis gastric cancer is urgently needed.

On the complex development of tumor initiation to metastasis, cancer cells need to adapt to permanently changing and often hostile environmental conditions. To survive in these conditions, tumor cells lose their epithelial feature and change to mesenchymal traits. This transition mainly expresses as losing cell-cell tight junctions, apical basal cell polarity and spindle-like cell shape [810]. Meanwhile with these changes, tumor cells show high migration, invasion, and survival features. These features are the core of tumor associated epithelial-mesenchymal transition (EMT). Therefore, EMT has became a highlight point in cancer treatment research.

EMT is influenced by several molecular networks. Various extracellular stimuli can activate or inhibit EMT process and most of them are classical growth factors which most secreted by tumor cells. Besides that, signaling pathways, such as TGF/BMP, Wnt, JAK-STAT, AP1 , Notch, NF- $\mathrm{kB}$ and Hippo signaling also induce or modulate the EMT process [11-13]. As a pivotal element in EMT process, bone morphogenetic proteins (BMPs) belonging to the transforming growth factor $-\beta$ (TGF- $\beta$ ) family binds to their receptors (BMPRs) followed by activating the phosphorylation and expression of small mothers against decapentaplegics 1/5/8 complex (SMAD1/5/8) in cell. Additionally, SMAD6, a protein that also belongs to small mothers against the decapentaplegic family, acts as a phosphorylated inhibitor playing a regulator role in BMP pathway $[14,15]$.Besides of SMAD family dependent working mode, several non-conventional BMP transducers have been identified, including KRAS, NOX and NRF2. With these transducers, BMP is able to serve as a co-modulator in reactive oxygen species (ROS), suggesting an indirectly influence on cancer progression through microsatellite instability status and chemoresistance [16-19]. Owing the modulation ability in cancer development, there are several research focusing on regulation mechanism of BMP pathway. Among these research, long non-coding RNA working through ceRNA mechanism on microRNA occupies a very special role [20-22].

Among various lncRNAs, FGD5-AS1 caught our attention for its influence on tumorigenesis and tumor development. According to sequences published on NONCODE (http://www.noncode.org/), we predicted putative proteins encoded by lncFGD5-AS1 using ORF Finder (https://www.ncbi.nlm.nih.gov/orffinder/) followed by analyzing the codon substitution frequency scores (CFS). The non-coding nature of lnc FGD5-AS1 was confirmed by no ORF was larger than $200 \mathrm{nt}$ and CFS was negative [23, 24]. Although role of FGD5-AS1 in oral cancer, periodontitis, glioma, and hepatocellular cancer has been fully investigated [25-28],. the function of FGD5-AS1 in gastric cancer still lacks enough evidence.

In this study, we firstly confirmed that lncFGD5-AS1 inhibited metastasis, proliferation and EMT in vitro and in vivo by regulating miR-196a-5p/SMAD6 to inhibit BMP pathway, which suggested the potential of FGD5AS1 as a candidate treatment target.

\section{Methods \\ Cell culture and cell transfection}

MKN74, MKN45 and HEK-293 cell lines were purchased from ATCC (American Type Culture Collection, Manassas, USA) though Genechem (Shanghai, China) with the cat.no. GCD01854, GDC01855 and GCD0156566. And all cells were grown in Roswell Park Memorial InstituteRPMI-1640 medium with 10\% fetal calf serum at $37^{\circ} \mathrm{C}$ and in a $5 \% \mathrm{CO} 2$ incubator. All cells were confirmed without any mycoplasma contamination tested by the Myco-Lumi ${ }^{\text {ma }}$ luminescence mycoplasma detection kit (C0298S, BiYunTian, China) annually. (The most recent detection was carried out on Aug 2020.)

Lentivirus particles were designed and purchased from Genechem (Shanghai, China), including LV-FGD5-AS1, LV-MiR-196a-5p-precursor, LV-anti-MiR-196a-5p, and controls. The vectors were as follows: Ubi-MCS-SV40EGFP-IRES-puromycin used for FGD5-AS1 overexpressed, hU6-MCSUbiquitin-EGFP-IRES-puromycin was used for MiR-196a-5p up-regulation, hU6-MCSCMVEGFP was used for MiR-196a-5p down-regulation. Lentivirus transfection was performed according to the manufacturer's instructions. Lentivirus was transfected with Enhance Liquid and Polyberene as ratio provided by Genechem.

Mimics of miR-196a-5p was designed and purchased from GenePharma (Shanghai, China). Mimics was transfected with lipo2000 (Invitrogen, ThermoFisher, USA).

The BMP pathway inhibitor, LDN-193189 from Axon Medchem (Netherlands), was dissolved with DMSO in $100 \mathrm{nM}$ and added into cells culturing in 6-well plates for $10 \mathrm{~h}$ before cell lysed for protein collection.

\section{Clinical and tissue samples}

The clinical tissue cDNA chip was purchased in Shanghai Outdo Biotech Co., Ltd. (CGt No: cDNAHStmA060CS01; Lot No:96*R100-M-201703xx-xx,), containing 30 pairs of cancer and para-cancer normal tissue. Para-cancer normal tissue was defined as the tissue more than $2 \mathrm{~cm}$ away but less than $3 \mathrm{~cm}$ away from the tumor edge. The 96-well plate was centrifuged before being performed with usage of SYBR Green real time PCR MasterMix (Takara (Japan)) and FGD5-AS1 primer. All the patients were gastric carcinoma diagnosed with pathological analysis. The tumor node 
metastasis (TNM) stage were assessed according AJCC Cancer Staging Manual [29].

\section{Cell migration assay}

Cell metastasis was examined using transwell assay, coculture chambers were purchased from BD Biosciences (San Jose, CA, USA). Serum-free medium are placed in top chambers, and medium containing 10\% FBS was added to the bottom chambers. Cells were evenly suspended in top chambers. Then the 6 -well plate with chambers were cultured for $24 \mathrm{~h}$ at $37^{\circ} \mathrm{C}$ with $5 \% \mathrm{CO} 2$. After incubation, the non-metastasis cells were gently removed from the top wells with a cotton-tipped swab and the chambers were fixed with methanol for $30 \mathrm{~min}$. The chambers were then stained with crystal violet for another $30 \mathrm{~min}$. Cell counting was facilitated by photographing the membrane through amicroscope (Zeiss) under $\mathrm{a} \times 10$ objective lens.

\section{Cell proliferation assay}

Cell counting Kit-8 (Genview, GK3607-100 T) was used to detect cell proliferation. 5000 suspended cells were planted in 96-well plate with complete medium containing $10 \% \mathrm{FBS}$. After the suspended cells adherence in $8 \mathrm{~h}$, $10 \%$ CCK solution was added in the well. Incubating for another $40 \mathrm{~min}$, then microplate reader was used to test the OD value at $450 \mathrm{~nm}$, which will indicate the proliferation of cells.

\section{Quantitative reverse transcription-PCR}

Trizol purchased from Invitrogen (Calsbad, USA) was used to extract total cell RNA as standard protocol. RNA concentration was tested by Nanodrop (Invitrogen, USA). PrimeScriptTM RT Master Mix kits were used to synthesis cDNA; and Taqman MirNA assay kit (designed for microRNA) and $\mathrm{SYBR}^{\bullet}$ Select Master Mix kit (designed for total RNA) were used for RT-qPCR analysis on Bio-Rad CFX96 qPCR instrument (Bio-Rad,Hercules, CA). All these kits were purchased from Takara (Japan). $\beta$-actin and U6 were used as reference genes.

\section{Western blot assay}

RIPA lysis (ThermoFisher, USA) was used for extracting total cell protein. Protein concentration was tested by BCA assay (ThermoFisher, USA). Equal amounts of protein were separated by SDS-PAGE gel as regular protocol. And the protein was transferred from gel to a PVDF membrane (Millipore). Then the PVDF membrane was blocked in 5\% BSA-TBST (Sigma, USA) for $2 \mathrm{~h}$ at room temperature, followed by primary antibody at 4-degree overnight. The membrane was washed $10 \mathrm{~min} 3$ times with TBST, followed by secondary antibody at room temperature for $1 \mathrm{~h}$. After another washing cycle, the membrane was visualized by ultra-sensitive ECL kit.
Results was calculated by Target protein/ $\beta$-actin based on band intensity tested by ImageJ.

The related antibodies used were as follows:

E-cadherin (CST, Boston, USA,14472), N-cadherin (CST, Boston, USA, 13116), Vimentin (CST, Boston, USA, 5741), Snail (CST, Boston, USA), MMP9(CST, Boston, USA), smad2/3((abcam, USA, ab202445), psmad2/3 (CST, Boston, USA),BMP4 (abcam, USA, ab124715),P-SMAD1/5/8(CST, Boston, USA, 13820), Anti-SMAD1/5/8 antibody (abcam, USA,ab80255), and TGF-BETA1 (abcam, USA, ab179695), SMAD6(SANTA CRUZ, USA, sc-25,321).

\section{Dual luciferase reporter assay}

Luciferase reporter assay. The binding sites of 3'UTR in SMAD6 were analyzed by Targetscan and were amplified by polymerase chain reaction (PCR) and inserted into the vector, which was designed and purchased by Genechem (Shanghai, China). Then transfected the 3'UTR plasmids and miR-196-5p mimics in to HEK 293 cells. Besides that, Renilla luciferase expression plasmid was co-transfected (Genechem Shanghai, China) as transfection control in all groups. After $24 \mathrm{~h}$, cells were lysed in $250 \mu \mathrm{l}$ of Passive Lysis Buffer (Promega) and $20 \mu \mathrm{l}$ were used to measure luciferase activity with the Luciferase Assay System (Promega). The different groups as follow: PGL3-NC + microup, PGL3-NC + microNC, PGL3-SMAD6MUT + micro up, PGL3-SMAD6MUT + micro NC, PGL3-SMAD6WT + micro up, PGL3$S M A D 6 \mathrm{WT}+$ microNC. Each group has 6 parallel holes, and the assay was repeated for 3 times.

\section{Fluorescence in situ hybridization (FISH)}

RNA-FISH was used to test location of lncRNA FGD5AS1. The florescence probes of FGD5-AS1 were purchased from Genechem (Shanghai, China). 18S rRNA was the probe for cytoplasmic control. For analysis the MKN-74 cells were cultured on slides. The slides were fixed in absolute ethyl alcohol for $15 \mathrm{~min}$ (Sigma), and then cells were treated by cold $0.1 \%$ Triton-100x for 15 min before hybridized with probes overnight at $37^{\circ} \mathrm{C}$. After washing with SSC/0.3\%Tween20 buffer, the coverslip was dyed with DAPI and fluorescence test was conducted with laser scanning confocal microscope (Leica Application Suite, Germany). DAPI channel was set at 410-485 nm showing the location of nucleic; Cy3 was chosen as cytoplasmic dye with excitation wavelength at 548-681 nm. The merged image was established with Leica Application. Scale bar was chosen as $50 \mu \mathrm{m}$ showing in the Fig. 2a.

\section{Tumor xenografts in mice}

Twelve 4-weeks old female J;NU Homozygous for Foxn1nu mice were obtained from the Medical 
Laboratory Animal Center of Xi'an Jiao Tong University and averagely separated into four cages randomly. The initial body weights of mice were $20 \pm 0.4 \mathrm{~g}$ (mean \pm STD). All nude mice were housed under specific pathogen-free conditions and the order of the cages changed every week by staff member to avoid position influence.

To test the function of lncFGD5-AS1 in vivo, we randomly separated these mice into lncFGD5-AS1 overexpressed group and control group ( 3 mice/cage, 2 cages/ group). The stable transfected Lv-FGD5-AS1 MKN45 and LV-control MKN45 cells were counted and suspended in $50 \%$ matrigel (USA, Corning) followed by injecting subcutaneously with 106 cells $0.1 \mathrm{ml}$ in each mouse. These mice were observed under standard SPF housing condition and the length, width and height of the tumor were tested every 3 days following randomly order for 5 weeks.

Before final test, two mice from control group were sacrificed for cachexia (severe body weight loss with body weight of $12.3 \mathrm{~g} \& 15.8 \mathrm{~g}$ ) at the early of 4th week; two mice from FGD5-AS1 overexpressed group were excluded from the experiment for the tumor didn't grow successfully. At final test time, each group had four 10weeks old mice. Mice were anesthetized with isoflurane while harvesting tumor tissue and sacrificed with cervical dislocation. The xenograft tumor tissues were harvested and weighted immediately followed by extracting protein and RNA from these tissues according to the common protocol. The protein and RNA were employed for further western blot and RT-QPCR test.

The experiment was in accordance with the regulation of the Ethics Committee of Xi'an Jiao Tong University and the Guide for the Care and Use of Laboratory Animals published by the US National Institutes of Health. The animal test was approved by the Ethics Committee of Xi'an Jiao Tong University and the ethics document was numbered as NO.G-271.

\section{Statistics methods}

The data in this article are shown as the means \pm SEM. Data was collected from at least three independent experiments. Paired Student-test was used to test the difference between paired groups. Unpaired Student-test was used to test the difference between un-paired groups. Chi-square test was used to test the difference for binary variables. Wilcoxon paired test was used to test differences between paired patients' samples because the difference value doesn't obey normal distribution. Graphpad (GraphPad Software, La Jolla, CA, USA) was used to do the statistics analysis. Image $J$ was used to collect intensity from western blot Fig. $P<0.05$ was marked as $* ; P<0.01$ was marked as ${ }^{* *} ; P<0.005$ was marked as ${ }^{* * * * *}, P<0.001$ was marked as $* * * * *$.

\section{Bioinformatics analysis}

The target gene and binding site of miR-196a-5p was predicted by TargetScan (http://www.targetscan.org/ vert_72/). The competition lncRNA and binding site of miR-196a-5p was predicted by Starbase (http://starbase. sysu.edu.cn/starbase2/index.php).

The Kaplan-Meier analysis was performed using the online Kaplan-Meier Plotter (http://www.kmplot.com) to estimate relapse-free survival curves of the 875 gastric cancer patients, and the median threshold was used as the cut-off point for the high and low groups of FGD5AS1 expression.

\section{Results}

\section{Lower level of IncRNA FGD5-AS1 indicated a worse prognosis}

To investigate the potential relationship between FGD5AS1 and the cancer process, we compared FGD5-AS1 levels in 30 patients' tumor tissue and para-cancer normal tissue. From Fig. 1a and Table 1, we can clearly summarize that cancer tissue had significantly lower FGD5-AS1 expression than normal tissue.

We separated all patients into high expression and low expression two groups by FGD5-AS1 expression levels in cancer tissue. The median expression level was regarded as the cut-off point. From Fig. 1 b\&c\&d\&e Fig. S1, we can summarize that lower expression of FGD5AS1 had strong relation with higher anatomic stage/ prognostic tissue and higher TNM stage. FGD5-AS1 influence moderately within the $\mathrm{M}$ stage, poorly within tumors that were $\geq 5 \mathrm{~cm}$ and differentiated degree. (Table 2 \& Fig. 1 b\&c \& d \& e) Besides that, an overall survival analysis based on FGD5-AS1 expression level was carried on in 875 gastric cancer patients (Data downloaded from TCGA database). As shown in Fig. 1f, higher FGD5-AS1 expression level had consistently higher survival probability from 0 months to 150 months (Log Rank $p=0.00045 ; \mathrm{HR}=0.66)$, which strongly indicated the positive relationship between FGD5-AS1 expression and a better prognosis.

In general, a lower expression of FGD5-AS1 strongly indicated worse prognosis and shorter survival time.

\section{LncRNA FGD5-AS1 mainly locates in the cell cytoplasm and combined with miR-196a-5p}

For positioning FGD5-AS1, we perform RNA-FISH in FGD5-AS1 highest expression cell line MKN-45. From Fig. 2a, we can see FGD5-AS1 labeled by red fluorescence mainly located in cytoplasm rather than nucleus, which indicates lncRNA FGD5-AS1 high likely acts through ceRNA theory. To support this point, we downloaded analyze of FGD5-AS1 location in various cell lines from LNCATLAS atabase (https://ncatlas.crg.eu/). Results also show FGD5-AS1 had a significantly higher 


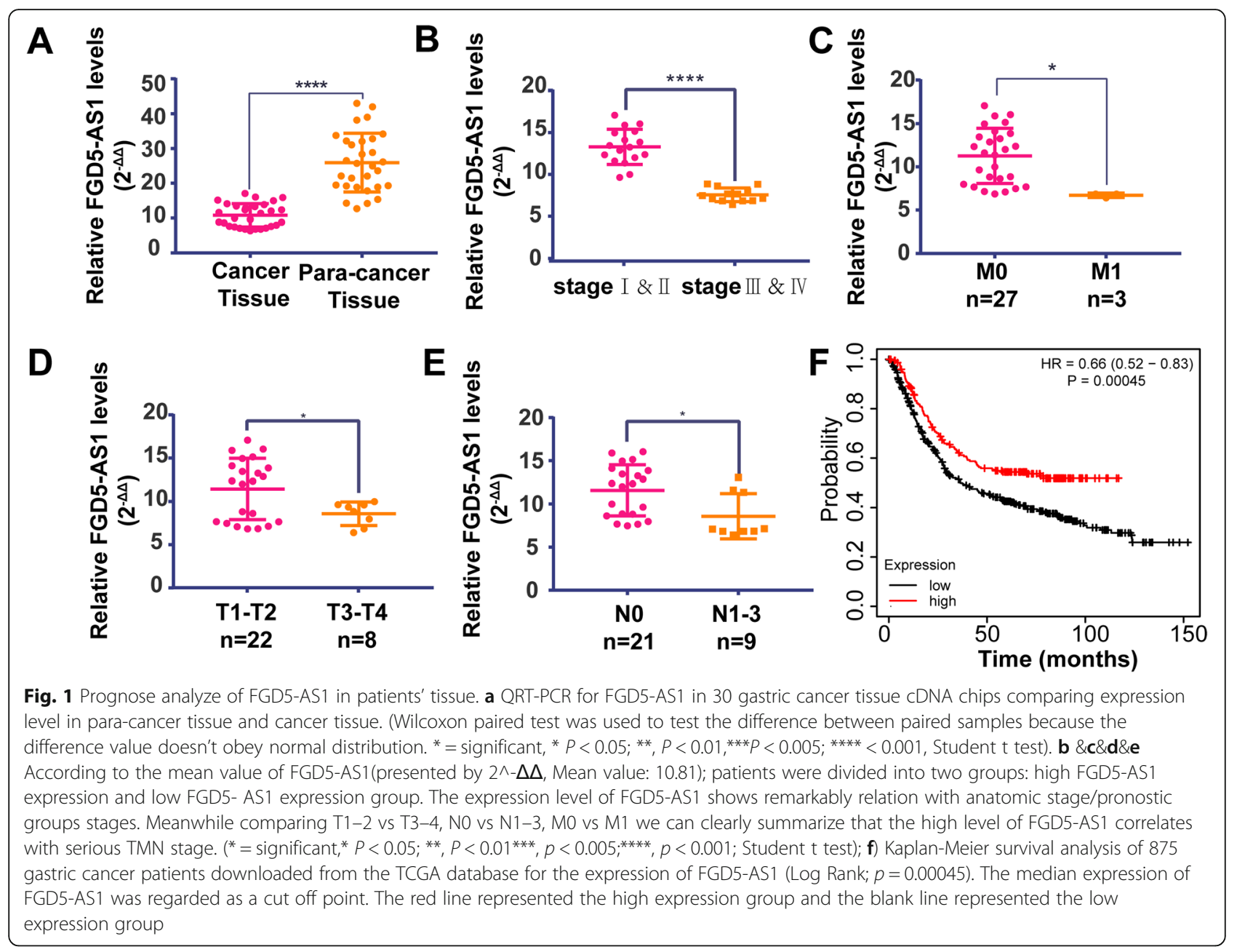

expression in cytoplasm. (Fig. S2) According to bioinformatics analysis results, miR-195a-5p high likely was the target gene of FGD5-AS1.To verify FGD5-AS1 and miR196a5p expression level correlation, we tested miR-196a$5 p$ in the same 30 patients' tumor tissue. As shown in Fig. 2c, there is an apparent negative trend between FGD5-AS1 and miR-196a-5p expression levels. To further test expression correlation between miR-196a-5p and FGD5-AS1, we overexpressed FGD5-AS1 in MKN45 and MKN-74 followed by testing miR-196a-5p mRNA level in these cell lines. (Fig. 2b\&e) The negative expression correlation strongly supported the ceRNA relationship between miR-196a-5p and FGD5-AS1.

Table 1 Expression of FGD5-AS1 in cancer and para-cancer tissue

\begin{tabular}{lllr}
\hline & Low expression & High expression & $\boldsymbol{P}$-value \\
\hline Para-cancer tissue & 6 & 24 & 0.0149 \\
Cancer tissue & 15 & 15 & \\
\hline
\end{tabular}

${ }^{a}$ The median expression level was used to distinguish high or low expression
Overexpressed FGD5-AS1 sharply inhibits cancer cells proliferation, migration and epithelial-mesenchymal transition process

Subsequently, we focused on FGD5-AS1 function in cancer metastasis and proliferation. We employed a transwell assay and a CCK- 8 assay to test its effects on cancer metastasis and proliferation, respectively. As illustrated by Fig. 3a\&b, migrated cells and OD/450 nm had sharply reduced in both MKN-45 and MKN-74 after overexpressing FGD5-AS1. In comparison, overexpressed FGD5-AS1 + miR-196a-5p mimics exhibited no differences with the control group. These results indicated that function of FGD5-AS1 can be rescued by increasing miR-196a-5p level, which strongly supported and indicated the ceRNA relationship and downstream role of miR-196a-5p. Based on the close relationship between epithelial-mesenchymal transition (EMT) and cancer initiation, we employed western blot and RTqPCR to test epithelial and mesenchymal markers. As shown in Fig. 3c-f, overexpressed FGD5-AS1 inhibited expression of mesenchymal markers ( $\mathrm{N}$-cadherin and Vimentin) and promoted expression of epithelial 
Table 2 Correlation of FGD5-AS1 expression with clinical and pathological characteristics of gastric cancer patients

\begin{tabular}{|c|c|c|c|c|}
\hline Variables & $\begin{array}{l}\text { Number of patients } \\
\text { (30) }\end{array}$ & $\begin{array}{l}\text { Low expression } \\
\text { (15) }\end{array}$ & $\begin{array}{l}\text { High expression } \\
\text { (15) }\end{array}$ & $P$-value \\
\hline \multicolumn{5}{|l|}{ Sex } \\
\hline Male & 24 & 12 & 12 & 0.99 \\
\hline Female & 6 & 3 & 3 & \\
\hline \multicolumn{5}{|l|}{ Age } \\
\hline$\leq 60$ & 18 & 8 & 10 & 0.4561 \\
\hline$>60$ & 12 & 7 & 5 & \\
\hline \multicolumn{5}{|l|}{ Tumor diameter } \\
\hline$<5 \mathrm{~cm}$ & 12 & 8 & 4 & 0.1360 \\
\hline$>5 \mathrm{~m}$ & 18 & 7 & 11 & \\
\hline \multicolumn{5}{|l|}{$\mathbf{T}$} \\
\hline $1-2$ & 8 & 1 & 7 & 0.0132 \\
\hline $3-4$ & 22 & 14 & 8 & \\
\hline \multicolumn{5}{|l|}{$\mathbf{N}$} \\
\hline 0 & 9 & 3 & 6 & 0.0053 \\
\hline $1-3$ & 21 & 12 & 9 & \\
\hline \multicolumn{5}{|l|}{ M } \\
\hline 0 & 27 & 12 & 15 & 0.0679 \\
\hline 1 & 3 & 3 & 0 & \\
\hline \multicolumn{5}{|l|}{ Serous membrane infiltration } \\
\hline No & 11 & 2 & 9 & 0.008 \\
\hline Yes & 19 & 13 & 6 & \\
\hline \multicolumn{5}{|l|}{ Differentiation degree } \\
\hline Moderately differentiated & 12 & 7 & 5 & 0.4561 \\
\hline Poorly differentiated & 18 & 8 & 10 & \\
\hline \multicolumn{5}{|c|}{ Anatomic stage/prognostic groups } \\
\hline $\mid \& \|$ & 13 & 3 & 10 & 0.0099 \\
\hline III\&IV & 17 & 12 & 5 & \\
\hline
\end{tabular}

${ }^{a}$ The median expression level was used to distinguish high or low expression

${ }^{\mathrm{b}} p$ value was calculated by chip-square test

markers (E-cadherin) with decrease of transcription factor SNAIL1 and migration marker MMP9.

In conclusion, reduction of mesenchymal markers and increase of epithelial makers indicated that a higher FGD5-AS1 induced tumor cells transiting from mesenchymal status into epithelial status, which will lead to cancer cell metastasis and proliferation.

\section{miR-196a-5p suppressed SMAD6/BMP pathway to influence EMT process}

To the exact mechanism of miR-196a-5p, we overexpressed and downregulated miR-196a-5p by lentivirus in MKN-74 and MKN-45, respectively. The overexpression and downregulation efficiency were shown in Fig. 4 a\&d.

Based on the bioinformatic analysis, SMAD6 is the most likely target gene of miR-196a-5p and shared the same combination sites with lncFGD5-AS1.
(Fig. 2d \& Fig. 4h) To verify the suppression of miR196a-5p on BMP pathway, expression of BMP4, tsmad1/5/8, p-smad1/5/8/ and smad6 were tested in miR-196a-5p overexpressed and downregulated cell lines by western blot and RT-PCR respectively. (Fig. 4 b, c, e, f, g) Results shown that SMAD6 exhibited a reverse trend with regulation of miR-196a-5p. Meanwhile, we employed dual-luciferase assay to confirm the combination between miR-196a-5p and between 3'UTR of Smad6. Predicted binding sites was cloned in luciferase-reported plasmid. After co-transfecting with miR-196a-5p mimics in smad6 wild-type sequences, the level of luciferase activity sharply reduced to $60.75 \%$ compared with the mutated binding sites. (Fig. 4h) Similar reduction of luciferase activity also shown in dual-luciferase report assay carried in GES-1. (Fig. S3). 


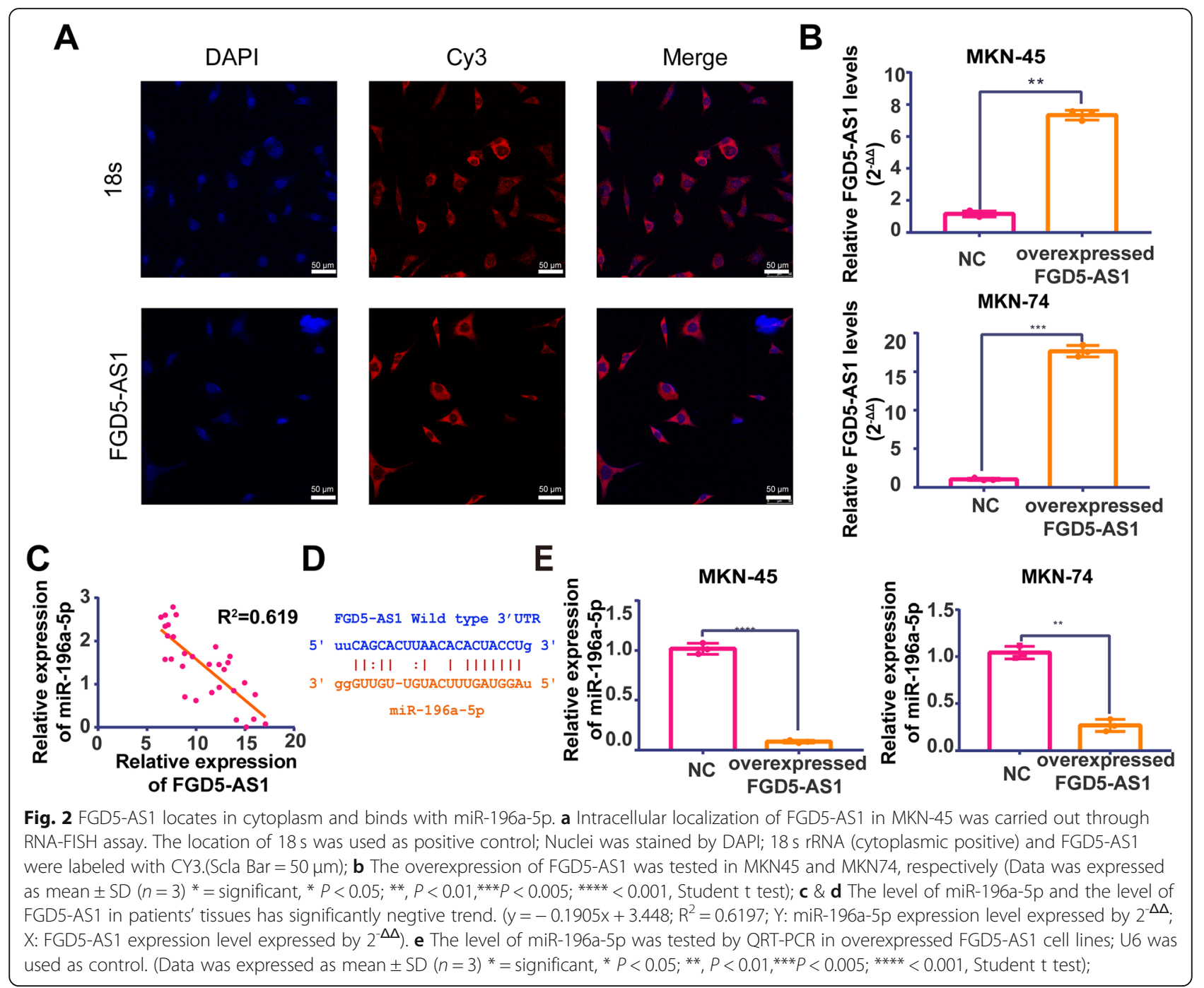

Besides that, the ratio of phosphorylated smad1/5/8 (p-smad1/5/8) and total smad1/5/8 (t-smad1/5/8) confirmed that the phosphorylation of BMP pathway influenced by miR-196a-5p. To further confirm the necessary role of the BMP pathway in the EMT inhibition process, we employed the whole BMP pathway inhibitor LDN193189 to rescue the inhibition. By comparing the first and third lane of Fig. 4i, overexpressing miR-196a$5 p$ sharply increased N-cadherin \& Vimentin level and decrease the E-cadherin level. In comparison, the overexpression of miR-196a-5p makes no influence on EMT markers in the blocking group. Surprisingly, the combination of BMP pathway block and miR-196a-5p overexpression resulted in a general increase of E-cadherin, which may be reasoned by other pathways influencing by microRNA and pathway block.

In general, miR-196a-5p combines with 3'UTR of SMAD6 to promote BMP pathway. A high level of FGD5-AS1 overwhelmingly combined with miR-196a-5p inducing overexpression of smad6 which inhibited the BMP pathway and further inhibited the EMT process.

\section{LncFGD5-AS1 suppressed tumor proliferation and EMT in vivo}

To examine the effect of LncRNA FGD5-AS1 on gastric cancer in vivo, we developed a nude mouse xenograft tumor model by subcutaneous injecting FGD5-AS1 overexpressing MKN45 and NC-MKN45. (Fig. 5a) QrtpCR showed that the tumor tissue in overexpression group had higher FG5-AS1 level and lower miR-196a-5p expression. (Fig. 5e \& f) After observing these tumors for 5 weeks, we found that the growth speed of tumor size in control group is higher than FGD5-AS1 group. (Fig. 5b) Meanwhile, the weight and final volume of harvested tumor tissue from mice in control group is heavier than FGD5-AS1 group. (Fig. 5c) In the 5th week, the tumor volume and tumor weight of the control group were $166.74+39.28 \mathrm{~mm}^{3}(n=4)$ and $2.78+0.57 \mathrm{~g}(n=4)$, 

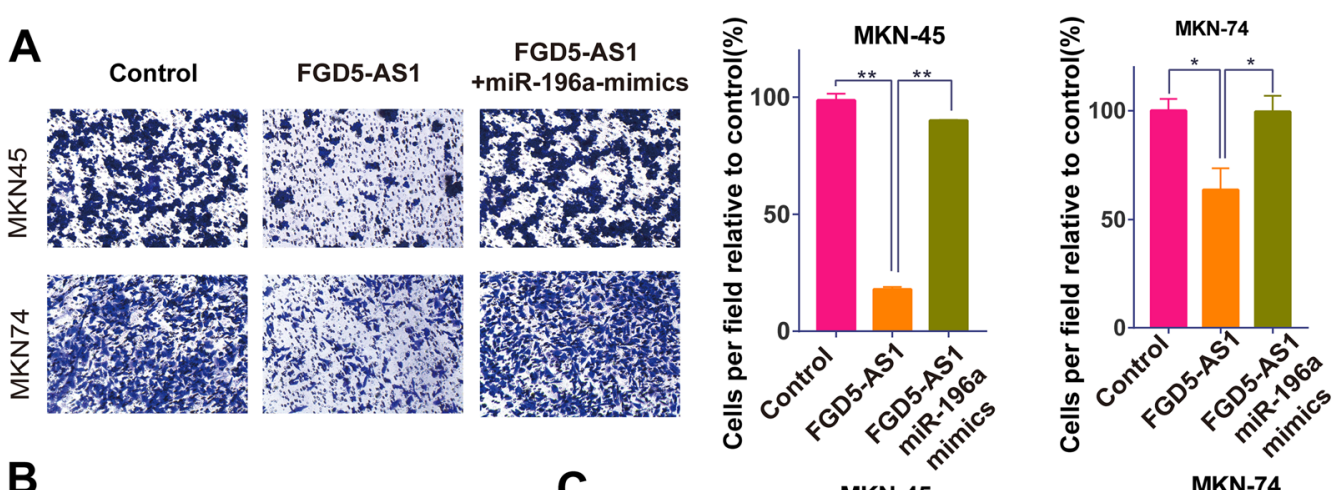

B

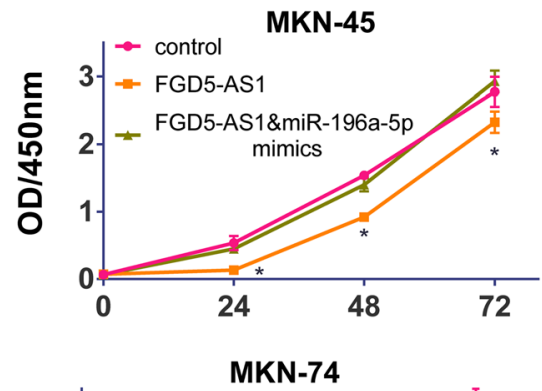

C

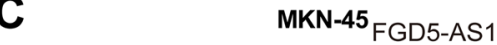

E cadherin Control FGD5-AS1 1 miR-196a

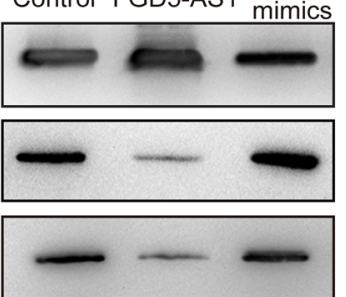

$\mathbf{N}$ cadherin

Vimentin

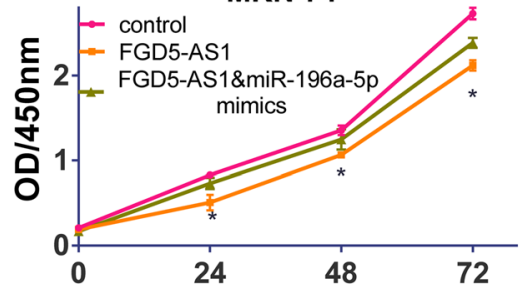

D

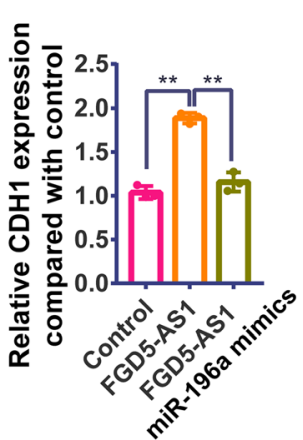

E
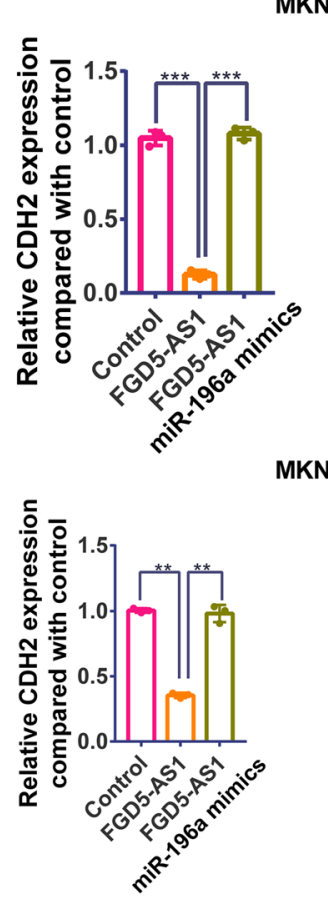

MMP9

SNAIL1

$\beta$-ACTIN
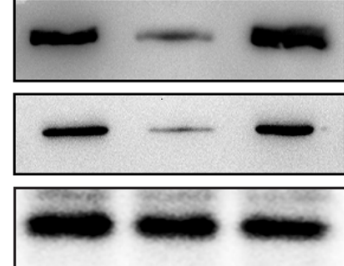

MKN-45

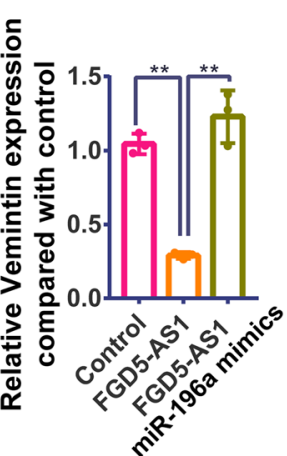

MKN-74

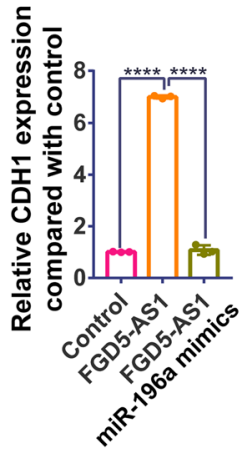

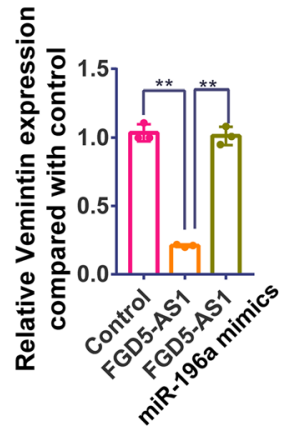
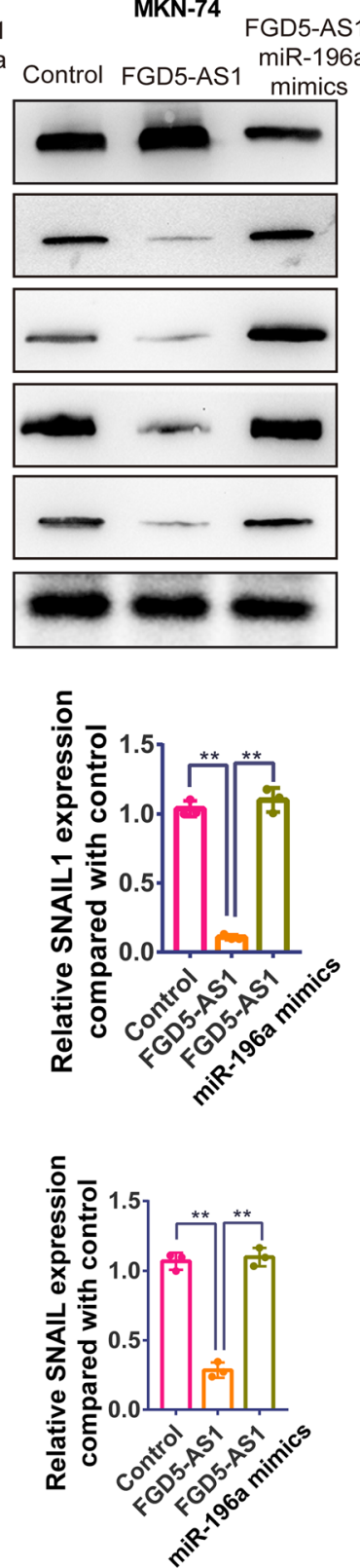

Fig. 3 (See legend on next page.) 


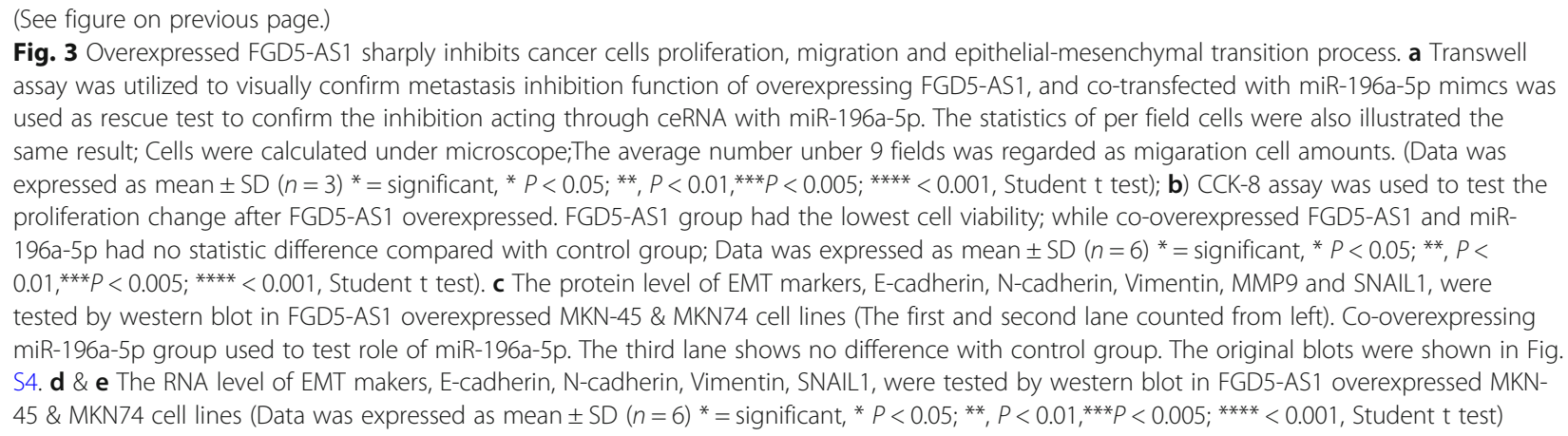

respectively. In contrast, the tumor volume and tumor weight of the FGD5-AS1 overexpressed group were $71.05+18.63 \mathrm{~mm}^{3}(n=4)$ and $1.18+0.39 \mathrm{~g}(n=4)$, respectively. Data were shown as mean \pm std.

To verify the function of the lncFGD5-AS1/miR-196a5p/SMAD6/BMP axis in vivo, we employed western blot and QRT-PCR in harvested tumor tissue. As shown in Fig. $5 \mathrm{~d} \& \mathrm{~g}$, the mRNA and protein levels of E-cadherin and SMAD6 were markedly increased with a decrease of $\mathrm{N}$-cadherin and BMP4 in xenograft tumors from the FGD5-AS1 group. Meanwhile, the ratio of $\mathrm{p}-\mathrm{smad1} / 5 / 8$ and $\mathrm{t}$-smad1/5/8 exhibits markedly decrease in FGD5AS1 group, which indicated the inhibition of BMP pathway.

\section{Discussion}

LncRNAs have been proved as an essential regulator and indicator in cancer. In this article, we found a high IncRNA FGD5-AS1 level in gastric cancer suppressing epithelial to mesenchymal transition and indicating a good prognosis. We clarified this conclusion through the analysis of patient samples, proliferation \& metastasis characteristics in cell lines, and tumor xenograft nude mice. Firstly, we found that more advanced levels of TNM stage, deeper serous membrane infiltration, higher anatomic stage/prognostic groups, and worse prognosis strongly related to lower expression of FGD5-AS1. Besides that, the overexpressed FGD5-AS1 group had less migration cells, lower cell viability, lower mesenchymal markers expression, and higher epithelia markers expression. To localize FGD5-AS1, we performed RNA-FISH in MKN-45 and further confirm it with the lncRNA location database. Unfortunately, limited by the access to proper patients' samples, we haven't performed RNAFISH locating FGD5-AS1 in biopsies samples. The cytoplasm location indicates FGD5-AS1 is likely working through a ceRNA mechanism. Subsequently, we employed a dual-luciferase report assay confirming the ceRNA relationship between FGD5-AS1 and miR-196a5p. Mimics of miR-196a-5p can rescue influence of FGD5-AS1 in vitro. In pathway level, FGD5-AS1 was confirmed working through rescuing the suppression of miR-196a-5p on SMAD6, BMP pathway inhibitor. As a regulator of oxidative factors, BMP4 is upregulated under oxidative stress which will facilitate cancer progression. The upregulated BMP4 is also able to reciprocally stimulate ROS pathway and act on cancer progression [14, 17, 30, 31]. Furthermore, tumor xenograft nude mice model proved that FGD5-AS1 could suppress tumor growth and EMT process through BMP pathway. The full schematic was shown in Fig. 6.

Prognostic markers could select patients who need more intensive treatment. Lacking proper prognostic markers is a common concern in gastric cancer treatment. Currently, clinical doctors prefer using symptom including tumour size, grade, and lymphovascular invasion to assess the patients' survival. However, gastric cancer is a highly heterogeneous disease indicating patients suffering similar symptom will have extremely different prognosis and survival status.

indicating that traditional evaluation system has remarkable limits in predicting patients' outcome [3-7]. To replace the conventional symptom prognosis evaluation system, extensive efforts have been dedicated to investigating non-coding RNA markers.

Our results strongly proved that higher FGD5-AS1 is a strong independent prognostic predictor of better survival. Interestingly, results provided by $\mathrm{Li}$ et al. [32] in their recent article showing that higher IncRNA FGD5-AS1 also performed a negative role in gastric cancer treatment through promoting chemoresistance. Similar differences also exist in difference tissue type, other research carried in colorectal cancer [33], lung cancer [34], and oral cancer [25]. The huge difference between these two kinds of conclusions illustrating the diversification in lncRNA function and heterogeneity in IncRNA expression [11, 35]. Mostly acting through the ceRNA mechanism, IncRNA directly working on the complementary microRNA followed by indirectly influencing the transcriptional expression level of downstream coding genes [36-38]. As an element in the dynamic network of non-coding 


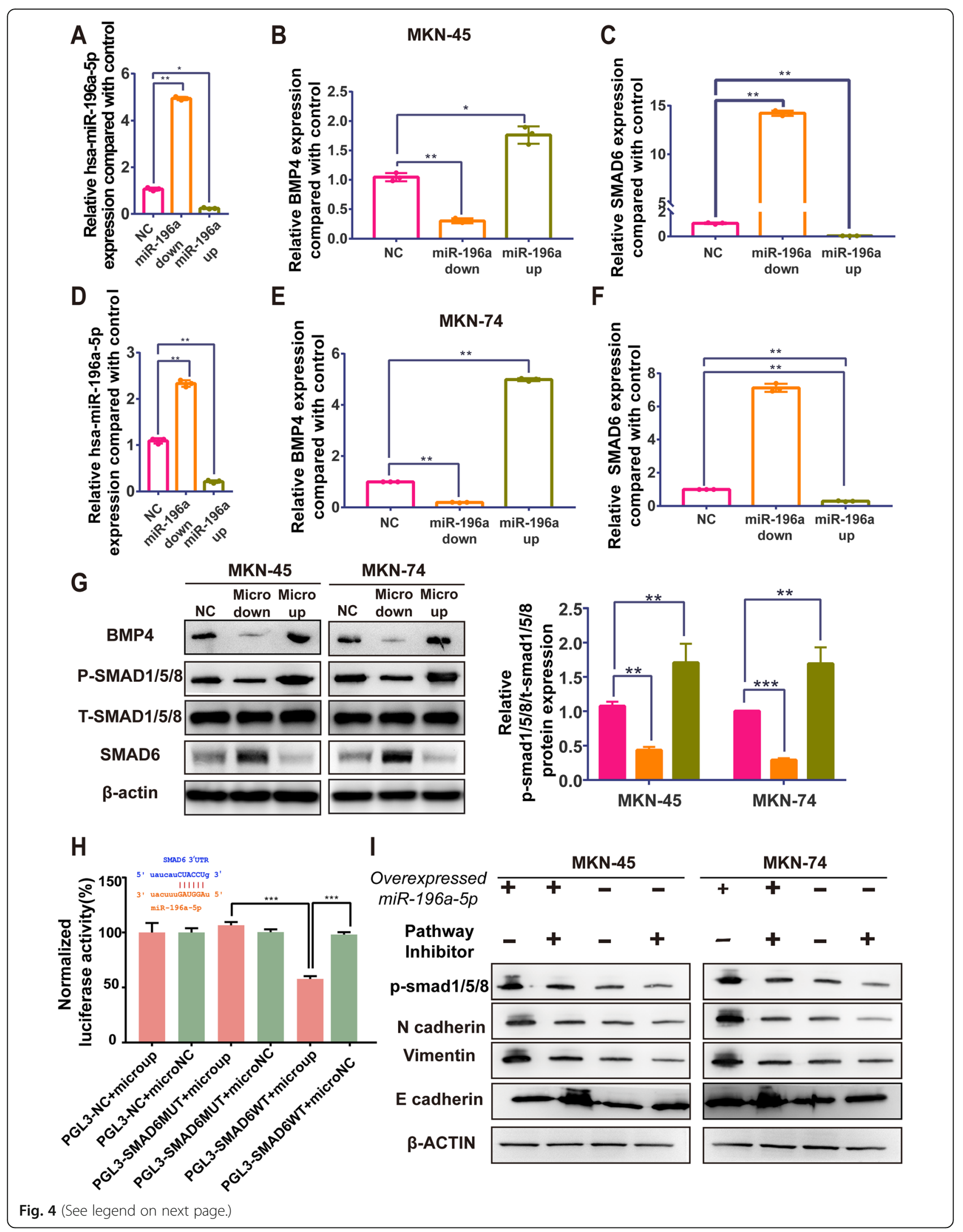


(See figure on previous page.)

Fig. 4 miR-196a-5p endocompetes with SMAD6 acting on BMP pathway to influence EMT process. a \& $\mathbf{d}$ The overexpression and downregulation of miR-196a-5p was tested in MKN45 and MKN74, respectively (Data was expressed as mean \pm SD $(n=3){ }^{*}=\operatorname{significant}^{*} P<0.05 ;{ }^{* *}, P<$ $0.01,{ }^{* * *} P<0.005 ;{ }^{* * *}<0.001$, Student $t$ test); b) \&c) \&e) \&f) After overexpressed and down regulated miR-196a-5p, the mRNA level of SMAD6 changes negtively with miR-196a-5p; while the mRNA level of BMP4 changes positively with miR-196a-5p. (Data was expressed as mean \pm SD $(n=3){ }^{*}=$ significant, $^{*} P<0.05 ;{ }^{* *}, P<0.01,{ }^{* *} P<0.005 ;{ }^{* * *}<0.001$, Student $t$ test $) ; \mathbf{g}$ Western blot for down and up regulated miR-196a-5 $p$ in MKN45 and MKN74 to test protein level of BMP pathway markers, BMP-4, t-smad1/5/8, p-smad1/5/8 and smad6. The figure shown that the level of BMP4 and p-smad1/5/8 changes positively with the level of miR-196a-5p. The ratio of p-smad1/5/8 and t-smad1/5/8 indicated that miR-196a$5 p$ influenced activation of BMP pathway. The original blots were shown in Fig. S5A \&S5B. $\mathbf{h}$ Dual-luciferase report assay. Wild-type and mutated 3'UTR-binding site was cloned in luciferase-reported plasmid and mimcs of miR-196a-5p or nc of miR-196a-5p was transferred with these plasmid into HEK293 cells. Compared with other groups, PGL3-SMAD6WT+ micro-up group normalize luciferase activity decrease by about 40\%. The significant reduce prove that the level of SMAD6 decrease when co-transfected with miR-196a-5p. (Data was expressed as mean \pm SD $(n=6) *=$ significant, ${ }^{*} P<0.05 ;{ }^{*}, P<0.01,{ }^{* * *} P<0.005 ;{ }^{* * *}<0.001$, Student $t$ test). i Effect of BMP pathway inhibitor LDN-193189. Western blot for EMT markers, E-cadherin, N-cadherin, Vimentin, and BMP pathway factors in MKN-45 and MKN-74 respectively as the following groups: micro-up, microup+LDN, microNC+DMSO and micro NC+ LDN. The original blots were shown in Fig. S6.

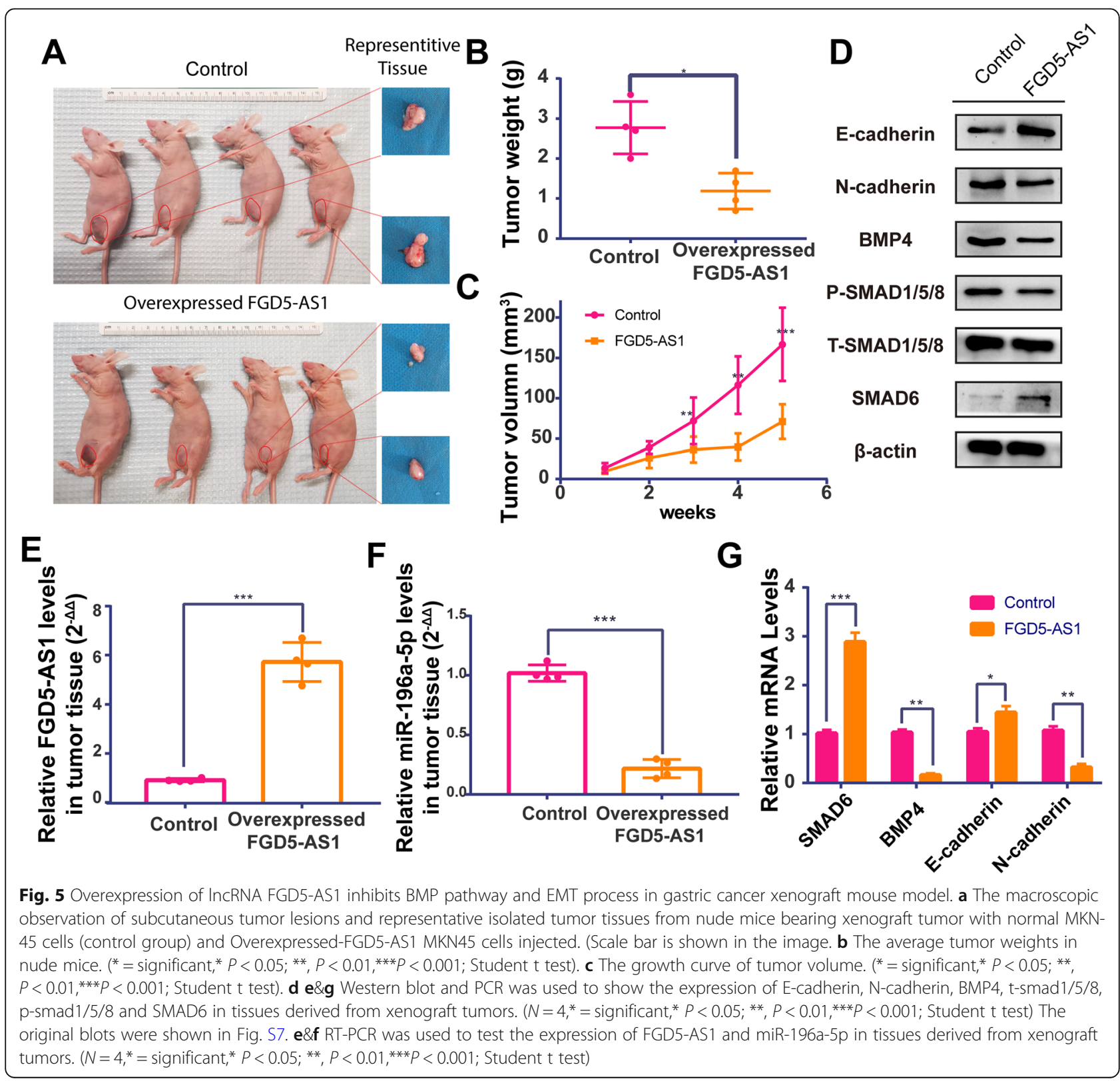




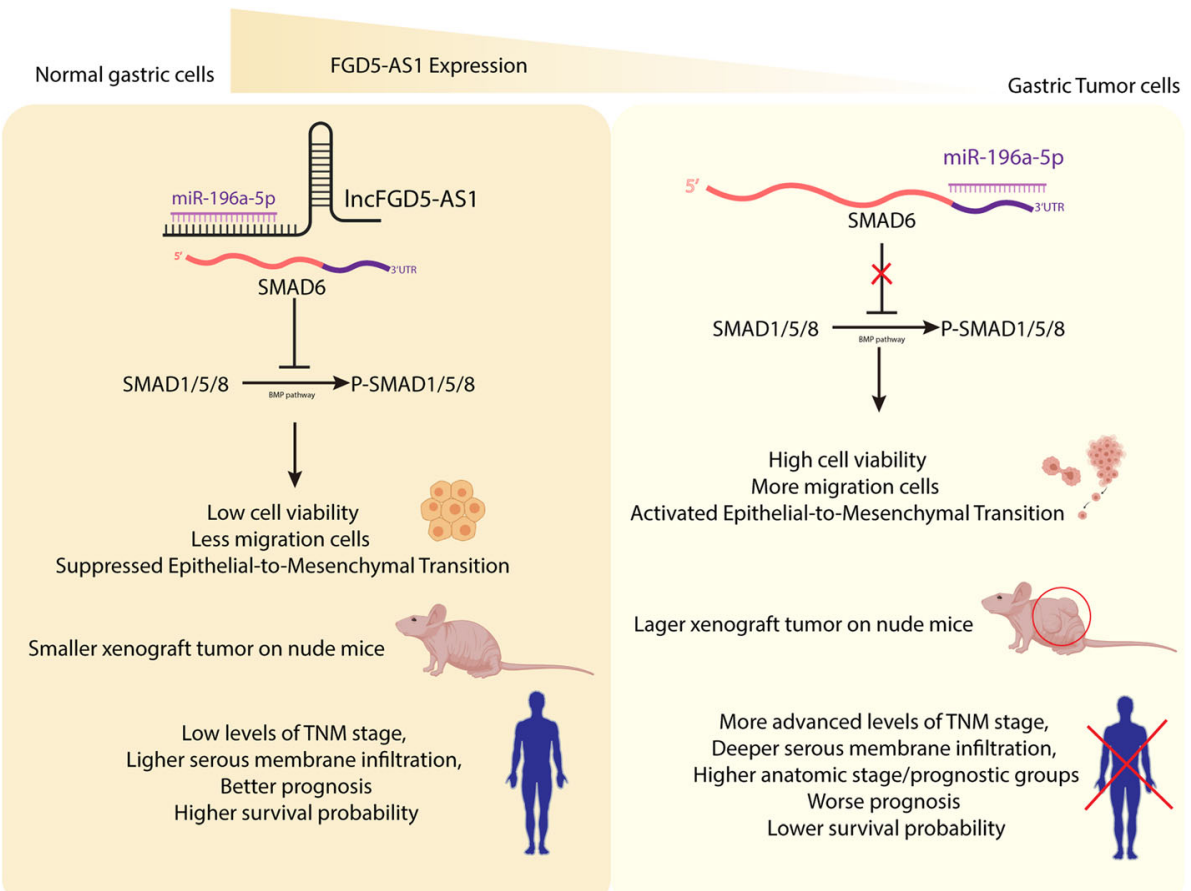

Fig. 6 Graphic Abstract. This research verified that a lower level of IncFGD5-AS1 could induce higher tumor cell viability and more migration tumor cells in vitro and larger tumor volume and tumor growth rate in vivo, which led to more advanced levels of TNM stage, deeper serous membrane infiltration, higher anatomic stage/prognostic groups, worse prognosis, and lower survival probability in gastric cancer patients. In mechanism, this article also investigated that IncFGD5-AS1 worked through regulating miR-196a-5p/SMAD6/BMP axis on the EMT process and served as a potential therapeutic candidate for gastric cancer. This figure was created with BioRender.com

RNA mechanism, lncRNA FGD5-AS1 owes bilateral function in tumor progression which requires more detailed research. Meanwhile, lncRNA have not been employed in clinical practice even though numerous previous researchers have focused on therapeutic potential of non-coding RNA [5, 6, 39, 40]. Their instability and tiny amount limited the accurate detection. Besides that, non-coding RNA has such wide influence in body which means it will have high sensitivity but extremely low specificity. The clear relationship between detectable abnormal value and typical clinical disease is still lack in clinical practice. Unfortunately, we have not solved these problems in this article. Nevertheless, we truly illustrated mechanism of lncRNA FGD5-AS1's function in gastric cancer. And we believe this will be basis for further clinical utilization of non-coding RNA.

\section{Conclusion}

In conclusion, as shown in the full schematic (Fig. 6) this research verified that a lower level of lncFGD5-AS1 could induce higher tumor cell viability and more migration tumor cells in vitro and larger tumor volume and tumor growth rate in vivo, which led to more advanced levels of TNM stage, deeper serous membrane infiltration, higher anatomic stage/prognostic groups, worse prognosis, and lower survival probability in gastric cancer patients.

In mechanism, this article also investigated that lncFGD5-AS1 worked through regulating miR-196a-5p/ SMAD6/BMP axis on the EMT process and served as a potential therapeutic candidate for gastric cancer.

\section{Abbreviations}

BMP: Bone morphogenetic proteins; BMPR: Bone morphogenetic proteins receptors; CeRNA: Competing endogenous RNA; CFS: Codon substitution frequency scores; EMT: Epithelial-to-mesenchymal transition; p-smad1/5/ 8: Phosphorylated small mothers against decapentaplegics $1 / 5 / 8$ complex; ROS: Reactive oxygen species; SPF: Specific pathogen-free; T-smad1/5/8: Total small mothers against decapentaplegics $1 / 5 / 8$ complex

\section{Supplementary Information}

The online version contains supplementary material available at https://doi. org/10.1186/s12885-021-08192-X.

\section{Additional file 1.}

Additional file 2.

Additional file 3.

Additional file 4.

Additional file 5.

Additional file 6 .

Additional file 7. 


\section{Acknowledgments}

This work was supported by Key Laboratory of Environment and Genes Related to Diseases, Ministry of Education.

\section{Authors' contributions}

L.L: Conceptualization, Methodology, Investigation, Validation, Writing Original Draft. C.Z: Validation, Investigation. J.Z.W: Formal analysis, Software, Writing - Review \& Editing. X.L: Visualization, Software. H.Y.Q: Visualization. G.J.Z: Resources. T.L: Resources. J.S.W: Supervision. J.Z: Writing - Review \& Editing, Funding acquisition. All authors have read and approved the manuscript.

\section{Funding}

This work is supported by correspondence author Jia Zhang through National Natural Science Foundation of China (No. 81702430), Fundamental Research Funds for the Central Universities (No. xij2018108) and Basic Research Program of Natural Science of Shaanxi Province (No.2018JQ8073).

\section{Availability of data and materials}

All data generated or analyzed during this study are included in this published article except for gastric cancer survival analysis data downloaded from the TCGA database. The datasets are available in the http://www. kmplot.com. LncRNA expression data was downloaded from https://www. ncbi.nlm.nih.gov/geo/ (GSE29272, GSE163988 and GSE158662).

\section{Declaration}

\section{Ethics approval and consent to participate}

The procedures were approved by the Ethics Committee of Xi'an Jiao Tong University and the ethics document was numbered as NO.G-271. The clinical tissue chip was purchased from Shanghai Outdo Biotech Co., Ltd. (CGt No: cDNA-HStmA060CS01; Lot No:96*R100-M-201703xx-Xx). All participates has approved the declarations.

\section{Consent for publication}

Not applicable.

\section{Competing interests}

The authors declare that the research was conducted in the absence of any commercial or financial relationships that could be construed as a potential conflict of interest.

\section{Author details}

'The Department of Thoracic Surgery, The First Affiliated Hospital of Xi'an Jiaotong University, Xi'an, Shaanxi, China. ${ }^{2}$ The Department of Radiology, The First Affiliated Hospital of Xi'an Jiaotong University, Xi'an, Shaanxi, China.

\section{Received: 13 August 2020 Accepted: 14 April 2021}

\section{Published online: 23 April 2021}

\section{References}

1. Chen W, et al. Cancer incidence and mortality in China, 2013. Cancer Lett. 2017;401:63-71. https://doi.org/10.1016/j.canlet.2017.04.024.

2. Shu P, et al. The IGCA staging system is more accurate than AJCC7 system in stratifying survival of patients with gastric cancer in stage III. BMC Cancer. 2017;17:238. https://doi.org/10.1186/s12885-017-3235-3.

3. Sano T, et al. Proposal of a new stage grouping of gastric cancer for TNM classification: international gastric Cancer association staging project. Gastric Cancer. 2017;20:217-25. https://doi.org/10.1007/s10120-016-0601-9.

4. Wu XJ, et al. Prognostic value of metastatic lymph node ratio as an additional tool to the TNM stage system in gastric cancer. Eur J Surg Oncol. 2015;41:927-33. https://doi.org/10.1016/j.ejso.2015.03.225.

5. Matsuoka T, Yashiro M. Biomarkers of gastric cancer: current topics and future perspective. World J Gastroenterol. 2018;24:2818-32. https://doi.org/1 0.3748/wjg.v24.i26.2818.

6. Liu HS, Xiao HS. MicroRNAs as potential biomarkers for gastric cancer. World J Gastroenterol. 2014;20:12007-17. https://doi.org/10.3748/wjg.v20.i34.12007.

7. Hartgrink HH, Jansen EP, van Grieken NC, van de Velde CJ. Gastric cancer. Lancet (London, England). 2009;374:477-90. https://doi.org/10.1016/s01406736(09)60617-6.
8. Polyak K, Weinberg RA. Transitions between epithelial and mesenchymal states: acquisition of malignant and stem cell traits. Nat Rev Cancer. 2009;9: 265-73. https://doi.org/10.1038/nrc2620.

9. Ye X, Weinberg RA. Epithelial-Mesenchymal plasticity: a central regulator of Cancer progression. Trends Cell Biol. 2015;25:675-86. https://doi.org/10.101 6/j.tcb.2015.07.012.

10. Valastyan S, Weinberg RA. Tumor metastasis: molecular insights and evolving paradigms. Cell. 2011;147:275-92. https://doi.org/10.1016/j.cell.2 011.09.024.

11. Cabili MN, et al. Integrative annotation of human large intergenic noncoding RNAs reveals global properties and specific subclasses. Genes Dev. 2011;25:1915-27. https://doi.org/10.1101/gad.17446611.

12. Ling $\mathrm{H}$, et al. Junk DNA and the long non-coding RNA twist in cancer genetics. Oncogene. 2015;34:5003-11. https://doi.org/10.1038/onc.2014.456.

13. Zhang A, Zhang J, Kaipainen A, Lucas JM, Yang H. Long non-coding RNA: a newly deciphered "code" in prostate cancer. Cancer Lett. 2016;375:323-30. https://doi.org/10.1016/j.canlet.2016.03.003.

14. Bragdon B, et al. Bone morphogenetic proteins: a critical review. Cell Signal. 2011;23:609-20. https://doi.org/10.1016/j.cellsig.2010.10.003

15. Wang RN, et al. Bone morphogenetic protein (BMP) signaling in development and human diseases. Genes Dis. 2014;1:87-105. https://doi. org/10.1016/j.gendis.2014.07.005.

16. Zhang YE. Non-smad signaling pathways of the TGF- $\beta$ family. Cold Spring Harb Perspect Biol. 2017;9. https://doi.org/10.1101/cshperspect.a022129.

17. Sánchez-de-Diego C, Valer JA, Pimenta-Lopes C, Rosa JL, Ventura F. Interplay between BMPs and Reactive Oxygen Species in Cell Signaling and Pathology. Biomolecules. 2019;9. https://doi.org/10.3390/biom9100534.

18. Polom K, et al. KRAS mutation in gastric Cancer and prognostication associated with microsatellite instability status. Pathol Oncol Res. 2019;25: 333-40. https://doi.org/10.1007/s12253-017-0348-6.

19. Sugimoto $R$, et al. Clinicopathological and molecular alterations in early gastric cancers with the microsatellite instability-high phenotype. Int J Cancer. 2016;138:1689-97. https://doi.org/10.1002/ijc.29916.

20. Yu J, et al. MicroRNA-182 targets SMAD7 to potentiate TGFß-induced epithelial-mesenchymal transition and metastasis of cancer cells. Nat Commun. 2016:7:13884. https://doi.org/10.1038/ncomms13884.

21. Liu Y, et al. Competitive endogenous RNA is an intrinsic component of EMT regulatory circuits and modulates EMT. Nat Commun. 2019;10:1637. https:// doi.org/10.1038/s41467-019-09649-1.

22. Grelet S, Link LA, Howley B, Obellianne C, Palanisamy V, Gangaraju VK, Dieh JA, Howe PH. A regulated PNUTS mRNA to IncRNA splice switch mediates EMT and tumour progression. Nat Cell Biol. 2017;19(9):1105-15. https://doi. org/10.1038/ncb3595.

23. Lin MF, Jungreis I, Kellis M. PhyloCSF: a comparative genomics method to distinguish protein coding and non-coding regions. Bioinformatics. 2011;27: i275-82. https://doi.org/10.1093/bioinformatics/btr209.

24. Mudge $J M$, et al. Discovery of high-confidence human protein-coding genes and exons by whole-genome PhyloCSF helps elucidate 118 GWAS loci. Genome Res. 2019;29:2073-87. https://doi.org/10.1101/gr.24 6462.118

25. Liu L, Zhan Y, Huang Y, Huang L. LncRNA FGD5-AS1 can be predicted AS therapeutic target in oral cancer. J Oral Pathol Med. 2020;49:243-52. https:// doi.org/10.1111/jop.12989.

26. Wu L, et al. FGD5-AS1 facilitates glioblastoma progression by activation of Wnt/ $\beta$-catenin signaling via regulating miR-129-5p/HNRNPK axis. Life Sci. 2020:117998. https://doi.org/10.1016/.lfs.2020.117998.

27. Sayad A, et al. Emerging role of long non-coding RNAs in the pathogenesis of periodontitis. Biomed Pharmacother. 2020;129:110362. https://doi.org/1 0.1016/j.biopha.2020.110362

28. Lin JZ, Lin N, Zhao WJ. Identification and validation of a six-IncRNA prognostic signature with its ceRNA networks and candidate drugs in lower-grade gliomas. Genomics. 2020;112:2990-3002. https://doi.org/10.101 6/j.ygeno.2020.05.016.

29. Washington K. 7th edition of the AJCC cancer staging manual: stomach Ann Surg Oncol. 2010;17:3077-9. https://doi.org/10.1245/s10434-010-1362-z.

30. Rider CC, Mulloy B. Bone morphogenetic protein and growth differentiation factor cytokine families and their protein antagonists. Biochem J. 2010;429: 1-12. https://doi.org/10.1042/bj20100305.

31. Mukhopadhyay S, et al. Undermining Glutaminolysis bolsters chemotherapy while NRF2 promotes Chemoresistance in KRAS-driven pancreatic cancers. Cancer Res. 2020;80:1630-43. https://doi.org/10.1158/0008-5472.can-19-1363. 
32. Gao Y, et al. Long non-coding RNA FGD5-AS1 regulates Cancer cell proliferation and Chemoresistance in gastric Cancer through miR-153-3p/ CITED2 Axis. Front Genet. 2020;11:715. https://doi.org/10.3389/fgene.2020. 00715.

33. Li D, et al. Long noncoding RNA FGD5-AS1 promotes colorectal cancer cell proliferation, migration, and invasion through upregulating CDCA7 via sponging miR-302e. In Vitro Cell Dev Biol Ani. 2019;55:577-85. https://doi. org/10.1007/s11626-019-00376-X.

34. Zhu F, Niu R, Shao X, Shao X. FGD5-AS1 promotes cisplatin resistance of human lung adenocarcinoma cell via the miR-142-5p/PD-L1 axis. Int J Mol Med. 2020. https://doi.org/10.3892/ijmm.2020.4816.

35. Gutschner T, Diederichs S. The hallmarks of cancer: a long non-coding RNA point of view. RNA Biol. 2012;9:703-19.

36. Kopp F, Mendell JT. Functional classification and experimental dissection of long noncoding RNAs. Cell. 2018;172:393-407. https://doi.org/10.1016/j.cell.2 018.01.011.

37. Boon RA, Jaé N, Holdt L, Dimmeler S. Long noncoding RNAs: from clinical genetics to therapeutic targets? J Am Coll Cardiol. 2016;67:1214-26. https:// doi.org/10.1016/j.jacc.2015.12.051.

38. Schmitz SU, Grote P, Herrmann BG. Mechanisms of long noncoding RNA function in development and disease. Cellular and molecular life sciences: CMLS. 2016;73:2491-509. https://doi.org/10.1007/s00018-016-2174-5.

39. Tang $W$, et al. CircRNA microarray profiling identifies a novel circulating biomarker for detection of gastric cancer. Mol Cancer. 2018;17:137. https:// doi.org/10.1186/s12943-018-0888-8.

40. Shekari N, Baradaran B, Shanehbandi D, Kazemi T. Circulating MicroRNAs: valuable biomarkers for the diagnosis and prognosis of gastric Cancer. Curr Med Chem. 2018;25:698-714. https://doi.org/10.2174/0929867324666171 003123425 .

\section{Publisher's Note}

Springer Nature remains neutral with regard to jurisdictional claims in published maps and institutional affiliations.

Ready to submit your research? Choose BMC and benefit from:

- fast, convenient online submission

- thorough peer review by experienced researchers in your field

- rapid publication on acceptance

- support for research data, including large and complex data types

- gold Open Access which fosters wider collaboration and increased citations

- maximum visibility for your research: over $100 \mathrm{M}$ website views per year

At $\mathrm{BMC}$, research is always in progress.

Learn more biomedcentral.com/submissions 